\title{
Delivery of Functionality in Complex Food Systems: Physically Inspired Approaches from Nanoscale to Microscale, Wageningen 18-21 October 2009
}

\author{
Erik van der Linden • Job Ubbink • Guus Duchateau
}

Received: 30 September 2010 / Accepted: 12 October 2010 / Published online: 27 October 2010

(C) The Author(s) 2010. This article is published with open access at Springerlink.com

\begin{abstract}
The Wageningen Delivery of Functionality symposium covered all aspects involved with food structural design to arrive at high-quality foods which meet demanding customer expectations and regulatory requirements. The symposium integrated aspects from the structural organization of foods at molecular and supramolecular scales to dedicated techniques required to describe and visualize such structures, the gastrointestinal events and how to model these in a laboratory setting, and finally the impact those food structures and ingredients have on the consumer's physiology and on the human perception. As an interdisciplinary platform, bringing together more than 160 researchers from academia and industry, the symposium meanwhile fulfills an important role in the food science community.
\end{abstract}

Keywords Symposium · Delivery · Functionality ·

Bio-accessibility $\cdot$ Food structure

E. van der Linden $(\bowtie)$

Food Physics Group, Wageningen University,

Bomenweg 2,

6703 HD Wageningen, The Netherlands

e-mail: Erik.vanderLinden@wur.nl

J. Ubbink

Food Concept and Physical Design,

Mühleweg 10,

CH-4112 Flüh, Switzerland

e-mail: job.ubbink@themill.ch

G. Duchateau

Unilever Research Vlaardingen,

Vlaardingen, The Netherlands

\section{Introduction}

This special issue of Food Biophysics journal contains papers originally presented at the 3rd International Symposium on Delivery in Complex Food Systems, which was held at Wageningen University, the Netherlands, from October 18 to 21,2009 . As the subtitle already indicates, the purpose of this symposium is to build a better understanding of the physical processes which are involved in creating food products and which impact their consumption, at all relevant length scales. These length scales bridge from molecular scale to microscale and finally to the macroscale. The topic is of interest to scientists from academia, industry and government as it relates to the building of basic understanding, to the production of highquality foods and to development and maintenance of the regulatory framework to protect consumers, the environment and ascertain a healthy competition in the food industry.

This symposium was the third one in what can truly be called a series now, with the first symposium held in Lausanne (CH), $2005^{1}$ and the 2nd one in 2007 in Amhurst (MA, USA). ${ }^{2}$ In this 3 rd symposium a number of talks concentrating on, or taking into account the fate, of common and newly designed food structures during their passage through the human gastro-intestinal tract. These steps target the actual delivery of the nutrients to the human body which is critical for its biological functionality. The focus of the symposium on delivery in the food context is obvious. Not only are consumers nowadays better informed about the relation between life-style and own health, of which their food consumption pattern is a critical part, there is also a strong development in the regulatory domain to protect the same consumers from misleading claims on food products. Foods with attached health claims which go beyond the normal 
nutritional content and impact, the so-called functional foods, have to meet all existing requirements and consumers expectations with regard to all sensory aspects, convenience and pricing, but also have to actually deliver the functional ingredient which is linked to the claimed health effect. Moreover the bioactivity of the added functional ingredient has to be demonstrated. The large number of dossiers submitted to the European authorities to assess the validity of functionality claims put forward by companies signals the great commercial interest in such products.

The symposium recognized five different sessions that are to be connected to address the issues above;

\section{Supramolecular Organization in Foods: Glassy Systems, Protein Assemblies, and Lipid Phases}

In regard to the preparation of foods, one has to meet boundary conditions on health, deliciousness, ingredient availability and cost. In addition, for both foods prepared at home and at industrial scale, flexibility in terms of formulation is additionally of importance. All these boundary conditions apply to one or more steps at the same time during the product life cycle: processing, transport, storage, consumption, and digestion.

There are two complementary ways to generate knowledge in this field. One is to investigate commonly existing foods on their function in terms of their microstructures and according constituents (molecular sized). The other is to prepare novel (micro-)structures and investigate these regarding their possible functions. The contributions in this session have to be viewed in terms of this latter area. One important class of ingredients is of course proteins, and their assembly is a key area within food development and production. In the session, two excellent contributions were presented in the area of protein/peptide assembly (by Professors Gazit, Hebrew University of Jerusalem, Israel, and Hammarstrom, Linkoping University, Sweden). In relation to the physics at larger length and time scales, an invited lecture was given by Professor Cipelletti, Université Montpellier II, on the long-time dynamics of microstructural evolution. This includes a discussion of promising experimental methods.

\section{Unraveling Food: Molecular Insights from Spectroscopy, Single-Molecule Techniques and Scanning Probe Microscopy}

The focus in this session was on how techniques and approaches developed in materials science, physics, and biophysics are having an impact in the field of food science and technology. Advances in measurement techniques from the single molecule to the mescopic level were highlighted, with emphasizes on the scientific framework in which their results can be used to advance our understanding of complex food systems.

\section{Biophysics of Nutrient Digestion and Absorption}

Understanding processes involved in delivery of the functional ingredient in the human GI tract is relatively new and was the focus of the Biophysics of nutrient digestion and absorption session. Better understanding of the processes involved methods to model or predict these in an experimental setting and finally meet delivery and regulatory requirements is essential for the functional food area. Not only will it help to substantiate the nutritional claims made for the food product, but products which score better on all these aspects are ultimately also the differentiator in the marketplace.

There is a whole cascade of events from food assembly, preparation, to digestive breakup starting in the mouth, to the complex stomach and intestinal events, involving biochemical processes to finally present the functional molecules and nutrients to the absorptive sites at the intestinal wall. This can be captured in the term bioaccessability and defines the amount of functional ingredient which is available for absorption.

So far, models which mimic the human gastro-intestinal events have been derived from anatomical and enzymatic understanding and less so from, e.g., a fluid dynamics approach. Certain process however are extreme dynamic and difficult to capture in in vitro models. This poses a challenge as for ethical, practical, cost constraint reasons, availability of in vitro models with good in vitro in vivo correlations would be ideal.

\section{Food Complexity: Physics, Physiology, and Perception}

In order to meet the challenges regarding the understanding of the development, production, consumption and digestion of foods one needs a multiscale approach. This means that one needs to connect many different scientific fields: physics, physiology, and the science of human perception. This session was organized as a first, exploratory attempt to bring together scientific developments in these various fields under the umbrella "complexity," which is a well-defined concept in many disciplines such as physics, network theory and sociology. We are convinced that in the food field the concept of complexity will have a major impact in the future, not only because of the complexity of, e.g., the physics or physiology, but in particular as the notion of complexity can help to modulate the way different scientific disciplines may interact and evolve together on a common topic, such as a food, food consumption or food digestion. 


\section{Food Development: Technologies and Ingredients to Face Nutritional Challenges}

For the first time in the symposium series, a session emphasizing the relation between food technology and nutrition was organized. In the session, examples were presented of successful products in which physical principles were utilized to create food products and food ingredients with enhanced stability, functionality and release of nutrients. In addition, in the session, conceptual approaches towards the development of new and sustainable food technologies were presented.

The conference was attended by about 160 scientists from academia and industry, and the formal and informal discussions were lively and stimulating, integrating old and new approaches. We are very pleased to announce that the next symposium, the 4th edition in this series, will be held in the early autumn of 2011 in Guelph, Canada.
Acknowledgements We thank the members of the Scientific Committee for their enthusiasm and their help in finalizing the program. Our special thanks are for Eva Oudshoorn for her excellent help and support in all practical aspects in the organization. We are very grateful to our sponsors (Danone Research, Friesland Campina, Unilever, VLAG graduate school of Wageningen University) for their financial support, which significantly facilitated the organization of the event.

Open Access This article is distributed under the terms of the Creative Commons Attribution Noncommercial License which permits any noncommercial use, distribution, and reproduction in any medium, provided the original author(s) and source are credited.

\section{References}

1. J. Ubbink, R. Mezzenga, Editorial: delivery of functionality in complex food systems: introduction. Trends Food Sci Technol 17 (5), 194-195 (2006)

2. D.J. McClements, Editorial: delivery of functionality in complex food systems; physically inspired approaches from nanoscale to microscale. Food Biophys 3, 111-112 (2008) 